\title{
Composition Assessment of a Power Distribution System with Optimal Dispatching of Distributed Generation
}

\author{
Muhammad Afzal1,2, Manuel S. Alvarez-Alvarado³, Zafar A. Khan,*, Mohammed \\ Alghassab ${ }^{5}$ \\ ${ }^{1}$ School of Engineering, University of Birmingham, Birmingham, United Kingdom \\ ${ }^{2}$ Faculty of Electrical Engineering, The University of Lahore, Lahore, Pakistan. \\ ${ }^{3}$ Faculty of Engineering in Electricity and Computing, Escuela Superior Politécnica del Litoral, Guayaquil, Ecuador \\ ${ }^{4}$ Department of Electrical Engineering, Mirpur University of Science and Technology (MUST), Mirpur A.K., Pakistan \\ ${ }^{5}$ Department of Electrical and Computer Engineering, Shaqra University, Riyadh, Kingdom of Saudi Arabia
}

\begin{abstract}
Increasing penetration of distributed generation (DG) is imminent in the new age of power distribution networks, which are smarter than the conventional grids. They enable the integration of DG into the power distribution network. This paper presents an assessment methodology for determining the optimal capacity and location of DG to ensure high reliability in a radial distribution network. The approach considers cost and the impact of aging on the DG and network topology for interconnection using genetic algorithm, which is a robust technique with wide solution space searchability and can potentially find global optima with fewer chances of getting trapped into local optima. A case study is simulated using three different scenarios to evaluate the impact of DG interconnection on the $13.8 \mathrm{kV}$ power distribution network. The scenarios comprise of situations without any DG, with DG interconnection and optimization of DG interconnection. The case study shows that the penetration of DG increases the reliability of the distribution network while reducing the expected energy not supplied (EENS). Although, the difference between EENS in the optimized DG integration and non-optimized DG integration is not very significant in a small network, however, it becomes apparent with the aging curve that optimized allocation of DG possesses significant benefits.
\end{abstract}

Keywords: Aging factor, Distribution generation, Genetic algorithm, Monte Carlo simulation, Photovoltaic system

Article History: Received: $8^{\text {th }}$ July 2020; Revised: 21 ${ }^{\text {st }}$ August 2020; Accepted: 26 ${ }^{\text {th }}$ August 2020; Available online: 27th August 2020

How to Cite This Article: Afzal, M., Alvarez-Alvarado, M.S., Khan, Z.A., and Alghassab, M. (2020) Composition Assessment of a Power Distribution System with Optimal Dispatching of Distributed Generation. International Journal of Renewable Energy Development, 9(3), 455-466 https://doi.org/10.14710/ijred.2020.31428

\section{Introduction}

Development of smart grids has enabled the integration of renewable energy sources as distributed generation (DG) at the power distribution network level. This development revolutionized the power system by changing the power generation regime from centralized to de-centralized generation. Integration of DG has affected the power system in terms of more complex system operations and maintenance. Integration of DG can potentially affect the system voltage control, load management and overall reliability of the system. Lack of consideration of the contributing factors such as DG integration can lead to power system blackouts in worst-case scenarios. To avoid such scenarios, consideration of studies using optimum integration of distributed generation has become imperative. In power system planning process, more attention is required to incorporate factors like technology mix, type, location and capacity of distributed generation resources. Particularly, integration of the DG is expected to bring new uncertainties in the power system. These uncertainties are expected to affect many important areas of power system and power system reliability. A brief discussion on the increasing penetration of DG and reliability evaluation is presented below.

Theoretical evaluations of market penetration potential and latest trends in photovoltaic applications are summarized in (Rújula et al 2005; Puttgen et al, 2003; Dellosa 2016; International Energy Agency, 2017; Premkumar et al 2018). Centralized photovoltaic (PV) is $70 \%$ of market and continuously expanding with competitive tenders, whereas China is leading global PV market with production and PV installations. Conventionally the reliability of power systems is evaluated using reliability test systems. For example, Reliability test system (RTS-96) is used as hybrid and typical reliability assessment system described in (Grigg, 1999) with production cost data and reliability indices. A sufficiently small educational reliability test system with

\footnotetext{
* Corresponding author: zafarakhan@ieee.org
} 
all main facilities like generation, transmission and radial distribution network is presented in ( $\mathrm{Li}, 2013)$.

However, reliability evaluation considering integration of DG presents different challenges. Technical problems associated with reliability of grid-connected PV systems are discussed in (Altamimi \& Jayaweera, 2017), (Eltawil \& Zhao, 2010) under climate changes and aging effects. Climate change produces inconsistent outputs over the years, whereas output power has constantly decreasing slop for whole life span of PV system as a result of aging factor. Quantitatively assessment is required for reliability of this challenging system.

Reliability of a power system is the combined reliability of individual components. Study on reliability evaluation of inverters in single-phase grid-connected PV systems is presented in (Calais et al 2002), whereas efficiency and accuracy of maximum power point trackers (MPPTs) are briefly discussed in (Jantsch et al 1997). It is concluded that PV system with efficient MPPT is more reliable as it works at maximum efficiency point. Some operating models of PV generators with reliability evaluation of active distribution networks are discussed in (Zhang et al 2013). Comparison of reliability performances of both central inverter and string inverter of grid connected PV systems is summarized in (Wang et al 2012).

A new stochastic model of PV system is proposed in (Zulu \& Jayaweera, 2014) to take in to account intermittent effects, transition states of PV system components and effects of insolation variations. Security and continuity of energy supply under dynamic thermal limits and variable weather conditions are analyzed in (Jayaweera \& Islam, 2014). Economic costs of outages especially in stressed distribution networks are highly dependent on combinatorial effects of variable weather and dynamic thermal limits. Change in weather conditions increases cost of outages and modelling of dynamic thermal limits decreases it.

Fault tree method is used for various parallel and sequential fault combinations of PV system in (Ahadi et al 2014) with the help of exponential probability distribution function. Reliability of system, maintenance planning and cost can be optimized by focusing on critical components. Functional modeling coupled with life cycle energy cost for maintenance and reliability of a PV system is given in (Stember et al, 1982). Components and reliability models of hybrid energy system consist of wind and photovoltaic system are studied in (Kishore \& Fernandez, 2011).

Integration of DG potentially affects the reliability of the grid due to the intermittency of the DG. Reliability of the grid can potentially be improved by increasing the dispatchable generation. However, another effective way of reliability enhancement is to take DG as separate system and more focus on strategically siting and sizing of DG with related economic aspects to increase the reliability of the system and economic benefits. In (Quezada et al 2006), various factors like DG penetration, concentration, technology mix, capacity factor, optimal location and reactive power control strategy are evaluated, which strongly affect energy losses associated with DG penetration.

A multi-objective evolutionary algorithm is developed in (Celli et al 2005) for siting and sizing of DG in distribution network. It uses mutual contradictive multi objectives functions such as cost of power losses, cost of energy, cost of network upgrading, and cost of energy not supplied to help planner in deciding a best compromise and trade-off between them. Genetic algorithm (GA) technique is used in (Borges \& Falcao, 2006) where optimal installations of DG are obtained by focusing on improvement of reliability, voltage profile and reduce electricity losses. Although, this method is applicable to multiple DG, it is not considering frequency related indices by assuming DG to be remained connected during fault. A comprehensive optimal strategy is proposed for the gradual replacement of diesel generators in an off-grid system with solar panels and batteries. Optimal sizing and siting reduces life cycle cost and initial investment by considering real life constraints. A trade-off between higher initial investment and system's lifetime saving is analyzed with time dependency of decision factors in (Rodríguez-Gallegos et al 2018). This robust multiobjective optimization is then extended to reduce levelled electricity cost, $\mathrm{CO}_{2}$ emissions and grid voltage deviation in (Rodríguez-Gallegos et al 2018). A similar approach is also employed to reduce total system cost while maintaining power quality and load demand in (Rodríguez-Gallegos et al 2017).

This paper presents an optimal composition assessment methodology incorporating reliability indices. The model considers aging factor and takes reliability test (RBT) system as case study as taken in (Billinton \& Li, 2013). Aging factor is modelled on PV system life span. The reliability of the system is strategically evaluated by optimal siting and sizing of DG using GA to reduce losses. Some indices are also introduced to evaluate their implications in power system reliability evaluation. Effects of important reliability indices are analyzed on the system as a whole with their effects on other indices to observe their individual trends. The proposed approach can be used for optimal siting and sizing of the DG integration to enhance the system reliability by considering different trends. These trends can be beneficial for control system attributes and the optimality of network.

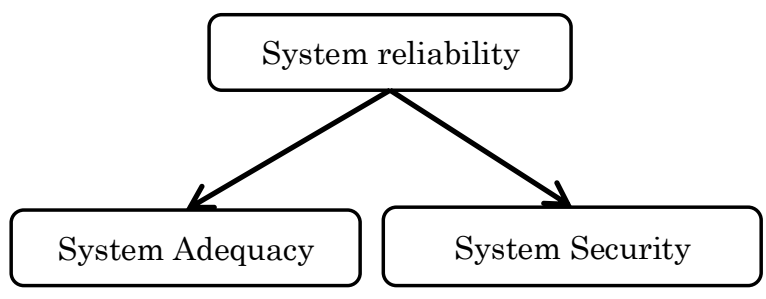

Fig. 1. Subdivision of system reliability (Billinton \& Li, 1994)

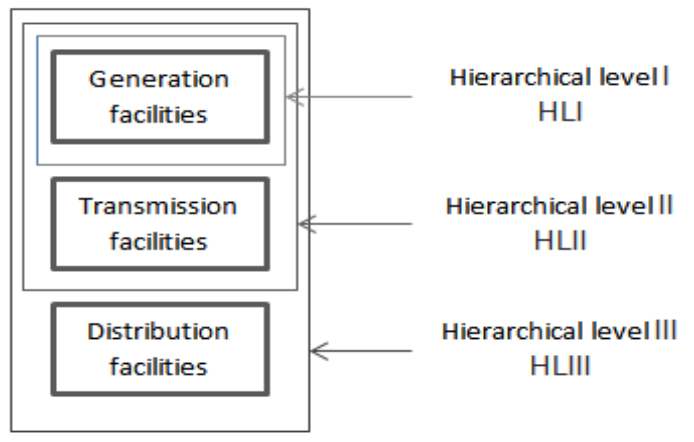

Fig. 2. Hierarchical levels of utility network (Billinton \& Li, 1994) 


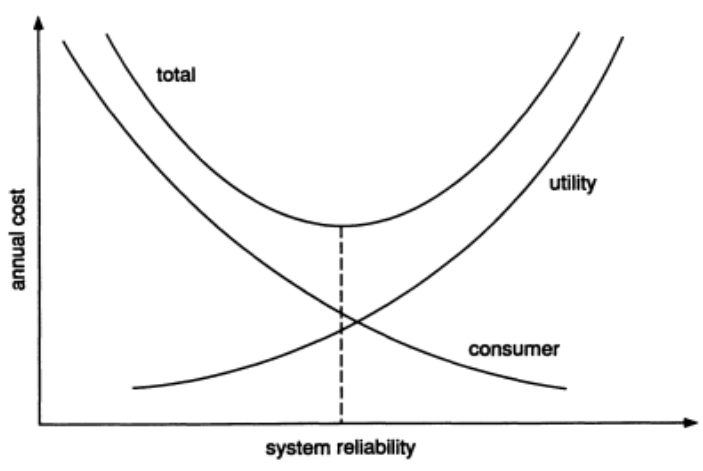

Fig. 3. System reliability as a controlling factor of consumer, utility and total cost (Billinton \& Li, 1994)

The rest of the paper is organized as follows: Section 2 discusses power system reliability in detail. Section 3 introduces reliability indices and Monte Carlo simulation. Section 4 presents introduction of important features of genetic algorithm. Section 5 presents the proposed methodology, whereas case study is discussed in section 6 . Finally, the conclusion is given in section 7 .

\section{System Reliability and Hierarchical Levels}

Probabilistic evaluation of power system reliability indices is combination of both severity and likelihood. System reliability has two basic facets, system adequacy and system security as shown in Fig 1 . System adequacy is the capability of system to satisfy all system constraints and load demands. System response to transient and dynamic disturbances is summarized as system security. For the purposes of system reliability evaluation, power system is often divided into generation, transmission and distribution zones. These zones can be combined in three hierarchical levels for adequacy study given in Fig.2.

Techniques for substation adequacy and radial distribution system reliability assessments are based on failure mode analysis including realistic failure and restoration processes. HL1 and HL2 failures have systemwide impacts, whereas HL3 failures have localized effects with frequent nature. A consistent appraisal of economics and reliability can be obtained by comparing adequacy cost or investment cost to adequacy worth or derived benefits as given in Fig. 3 and taken from (Billinton \& Li, 1994).

\section{Distribution System}

Evaluation of distribution system adequacy needs some suitable adequacy indices for assessment at actual load points. Overall evaluation of HL3 or distribution system can be very complex in most systems involving all functional zones starting from generation to individual load points. Analysis of radial distribution system is based on failure mode analysis involving realistic failures and restoration processes (Billinton \& Li, 1994). Reliability evaluation using the standard reliability assessment indices and the impact of PV aging is as follows:

\subsection{Reliability Indices}

Adequacy of an individual customer is best reflected by HL3 or distribution system indices (Billinton \& Li, 1994). Some important indices for distribution system adequacy assessment are:

a) SAIFI stands for system average interruption frequency index and unit is interruptions/system customer/yr.

$$
S A I F I=\frac{\sum_{i \in R} \lambda_{i} N_{i}}{\sum_{i \in R} N_{i}}
$$

where load point $i$ has $N_{i}$ numbers of customers and $\lambda_{i}$ failure rate. $R$ represents the total load points included in the reliability evaluation (Billinton \& Li, 1994).

b) SAIDI stands for system average interruption duration index having unit of hr/system customer/yr.

$$
S A I D I=\frac{\sum_{i \epsilon R} U_{i} N_{i}}{\sum_{i \in R} N_{i}}
$$

where annual unavailability or outage time at load point $i$ is represented by $U_{i}$ given in hours per year (Billinton \& Li, 1994).

c) CAIDI stands for customer average interruption duration index having unit of hour per customer interruption (Billinton \& Li, 1994).

$$
C A I D I=\frac{S A I D I}{S A I F I}=\frac{\sum_{i \in R} U_{i} N_{i}}{\sum_{i \in R} \lambda_{i} N_{i}}
$$

d) $S A I F I-R I$ stands for SAIFI reduction index. It is ratio of $S A I F I$ values after DG and before DG. It is unit less index.

$$
\text { SAIFI }-R I=\frac{\text { SAIFI after } D G}{\text { SAIFI before } D G}
$$

e) SAIDI-RI stands for SAIDI reduction index. It is ratio of SAIDI values after DG and before DG. It is unit less index.

$$
S A I D I-R I=\frac{\text { SAIDI after } D G}{\text { SAIDI before } D G}
$$

f) SOI stands for system outage index. It is ratio of total annual supply outage hours after DG and before DG. It is unit less index.

$$
S O I=\frac{(S A I F I * S A I D I)_{\text {after } D G}}{(S A I F I * S A I D I)_{\text {before } D G}}
$$

\subsection{Aging Effects on PV}

Life of PV modules can be as high as 25 years. Manufactures give warranty of at least $90 \%$ output power after $10-12$ years and $80 \%$ output power after $20-25$ years. Tolerance can be assumed as 5\%. Linear degradation of PV output power is given in (7) and (8) and taken from (Altamimi \& Jayaweera, 2017).

$$
\begin{gathered}
P_{t}=P_{\text {ini }}[1-(k-L) d] \\
P_{P V, k}=-P_{t}+2 P_{i n i}
\end{gathered}
$$

where $k=1,2,3 \ldots L, P_{t}$ is total power of PV, $P_{i n i}$ is initial power, $\mathrm{k}$ is specific year, $\mathrm{L}$ is life span of $\mathrm{PV}$ and $\mathrm{d}$ is constant slop. 
Usually, adequacy and reliability studies in power system use Monte Carlo Simulations, which is the process that uses random number for calculation of the structure of a stochastic process. It is commonly used in many stochastic processes such as power system reliability assessment, where the method estimates the indices by simulating the actual process and random behavior of the system states (Billinton \& Li, 1994). The method simulates the process by treating it as a series of experiments, which are often referred to as the Monte Carlo simulation.

\section{Optimization-Genetic Algorithm}

Heuristic methods are intelligent as they move from one solution to another solution using human reasoning rules. The heuristic algorithm search solutions inside a subspace of total space so they can give a good solution in reasonable computing time. The most important advantage of them is that they are not dependent on restrictive assumptions like objective function derivative existence and continuity etc. Some heuristic methods are Tabu Search method (TS), Simulated Annealing (SA) and Genetic Algorithms (GAs). Each one has its own advantages and drawbacks. The TS is a deterministic method and search cannot be restricted in a set of solutions by any random process. SA needs some parameters difficult to determine and takes long computation time. Genetic Algorithms are based on natural selection, independent of initial configuration choice and produce high quality solutions (Gerbex et al 2001). Genetic algorithm is widely used optimization technique and it finds applications in different areas including power systems (Goyal et al 2011).

Genetic algorithm is a search algorithm based on natural selection and the fittest survival process. It is a robust method as no restriction on solution space is imposed during the process. The strength of the method is to use the information structures from past solution guesses to improve performances of the future solution structures. It maintains a constant population size of solution structure throughout process so entire solution space is considered and multiple solutions are detected (Walters \& Sheble, 1993).

Genetic algorithm has many optimization applications as it is efficient and flexible. Advantages of this technique are using stochastic operators and searching for multiple optimum solutions parallel (Chen \& Chang, 1995). As it evaluates many points simultaneously without assuming search space continuous or differentiable so most likely it converges to the global solution. Information is exchanged between peaks and ending at the global optimum.

It is more flexible than other search methods as it only requires information regarding the quality of solution given by a set of each parameter and not complete information such as derivative information for problem parameters and structure. Some important differences of genetic algorithm and other traditional searching algorithms can be summarized as (Bouktir et al, 2004):

- Genetic algorithm works with a population string instead of a single point and searches many peaks parallel.
- It works directly with characters string representing parameters set instead of parameters themselves.

- It uses probabilistic methods as compared to deterministic methods.

- It uses objective function information in contrast to using derivative knowledge.

- It has the ability to find out solutions simultaneously in different areas.

\section{a. Procedure}

A simple Genetic Algorithm is an iterative process, which keeps population size constant in each iteration. Three genetic operators, reproduction, crossover and mutation are performed in each step to generate the new population. Chromosomes of the new population are evaluated through fitness function value and better candidate solution is generated. A simple Genetic Algorithm procedure can be summarized as (Bouktir et al 2004):

1. A random population is generated

2. Fitness function value is evaluated for each string of population

3. Offspring strings are produced through reproduction, crossover and mutation.

4. Evaluate fitness function values for new strings.

5. If a fitness function goal is achieved and other search requirements are fulfilled then best chromosome or string will be solution. Otherwise go to step 3 and repeat these steps until final goal is achieved.

\section{b. Chromosomes Encoding and Decoding}

Genetic Algorithm works with strings and not with parameters themselves. Binary coding is used for this purpose. Encoding is a process to convey important information by representing a solution as string where each bit of a string represents a characteristic of solution. Decoding is a reverse procedure (Bouktir et al 2004), (Bodenhofer, 2003).

\section{c. Crossover}

Crossover is the primary genetic operator, which explores new regions of search space. A crossover point is selected randomly and segments of selected parent strings on left side of this point are switched to produce offspring (Bouktir et al 2004).

\section{d. Mutation}

Mutation is the secondary operator and prevents local solution. Mutation is change of random bit value, which has less probability of change (Bouktir et al 2004).

\section{e. Reproduction}

Reproduction is based on natural principle of survival of fittest one. Score of solution increases if it is closer to optimal solution and it will have more copies. However, total number of solutions remains constant (Bouktir et al 2004). 


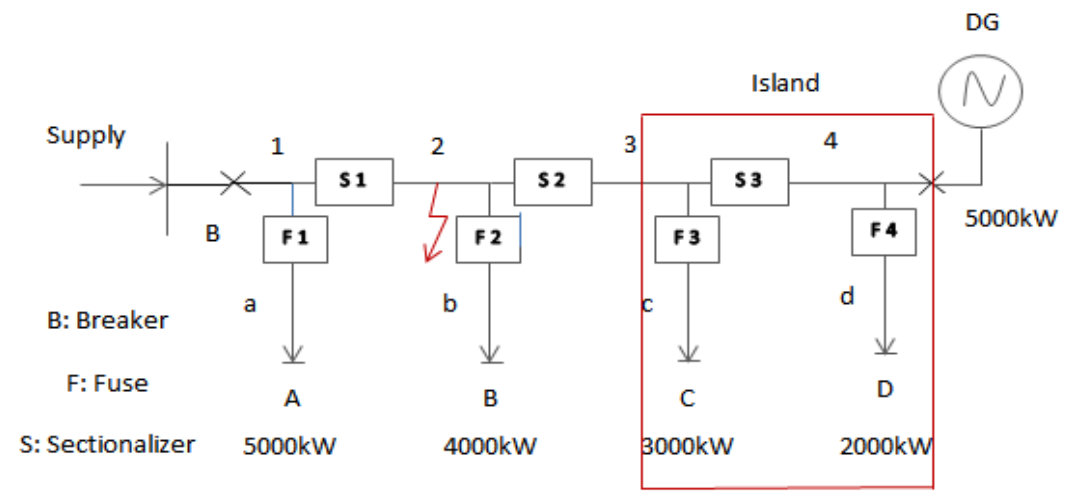

Fig. 4. Reliability benefits of DG

\section{f. Fitness Function}

Fitness function quantifies optimality of a solution against all solutions depending on that how close it is to optimal solution (Bodenhofer, 2003).

\section{Methodology}

The methodology presented in this paper is based upon the number of candidate locations for DG allocation as well as a list of candidate DG units. Investment and technical information for candidate DG units help in finding an optimal solution for the system. The optimal solution must have high fitness function value, which results in a reduction of system losses and minimum cost. The number and size of DG units are decided by desired penetration level, which in our case is determined by total loads provided by DG.

The proposed methodology is applied to a system taken from RBT system (Li, 2013) as shown in Fig. 4. The test system and related information are detailed in $(\mathrm{Li}$, 2013). A fault is assumed to occur on section 2 and as a result, it is isolated by opening isolator on section 3, whereas loads C and D are supplied by installed DG units. Network topology and load demands are considered constant in the planning period of this topology.

\section{a. Fitness Function}

Genetic Algorithm uses fitness function value to find the optimal solution. Fitness function value quantifies the optimality of the solution. Here fitness function is evaluated through benefits obtained by DG installations in the form of the reduction in losses and cost benefits.

$$
F=\frac{\text { Benefits }(B)}{\text { Total } \operatorname{Cost}(C)}
$$

where

$$
\begin{aligned}
\text { Benefits }(B)= & (\text { losses before } D G \text { installation } \\
& - \text { losses after } D G \quad \text { installation }) \\
& \times \text { cost } / k W \\
\text { Total Cost }(C)= & \text { Installation cost }+ \text { investment cost } \\
& + \text { maintenance cost }
\end{aligned}
$$

\section{b. Constraints}

Some constraints are imposed to find out optimal solution. They can be summarized as

- Reliability indices should be better after DG installation and remain in specified limits.

- Total DG penetration must not be greater than specified amount of power.

\section{c. Parameters}

Parameters used for Genetic Algorithm to implement proposed methodology can be summarized as (Borges \& Falcao, 2006):

- Population set size: 50

- Total number of generations: 30

- Cross over operator probability: $95 \%$

- Mutation operator probability:5\%

- Tournament selection type

- One point crossover

- Constant mutation type

\section{d. Encoding and decoding}

Coding and decoding of solution are important step in genetic algorithm. An optimal solution is optimal size of DG at candidate locals. Each solution is coded as a vector whose length is equal to number of candidate locals and value is installed capacity of DG.

Each chromosome value is an integer type $\{0,1,2,3 \ldots\}$, where integer 0 means no DG is installed, whereas any other number represents sequentially a DG type from Table 1. Decoding is translating integer number in respective DG type. An example of coding and decoding for two candidate locals and four DG types (DG_A-DG_D) is shown in Fig. 5.

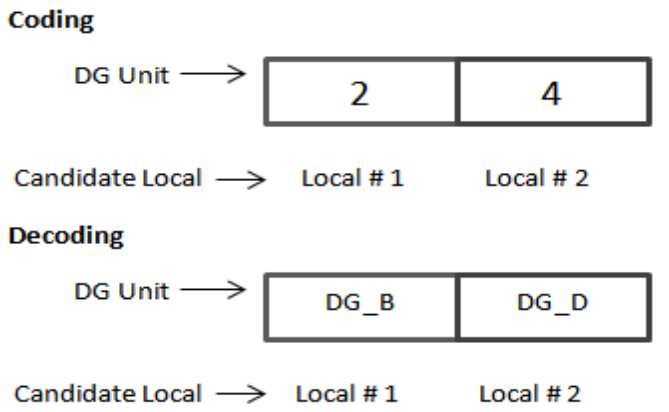

Fig. 5. Coding and decoding of chromosome (Borges \& Falcao, 2006) 


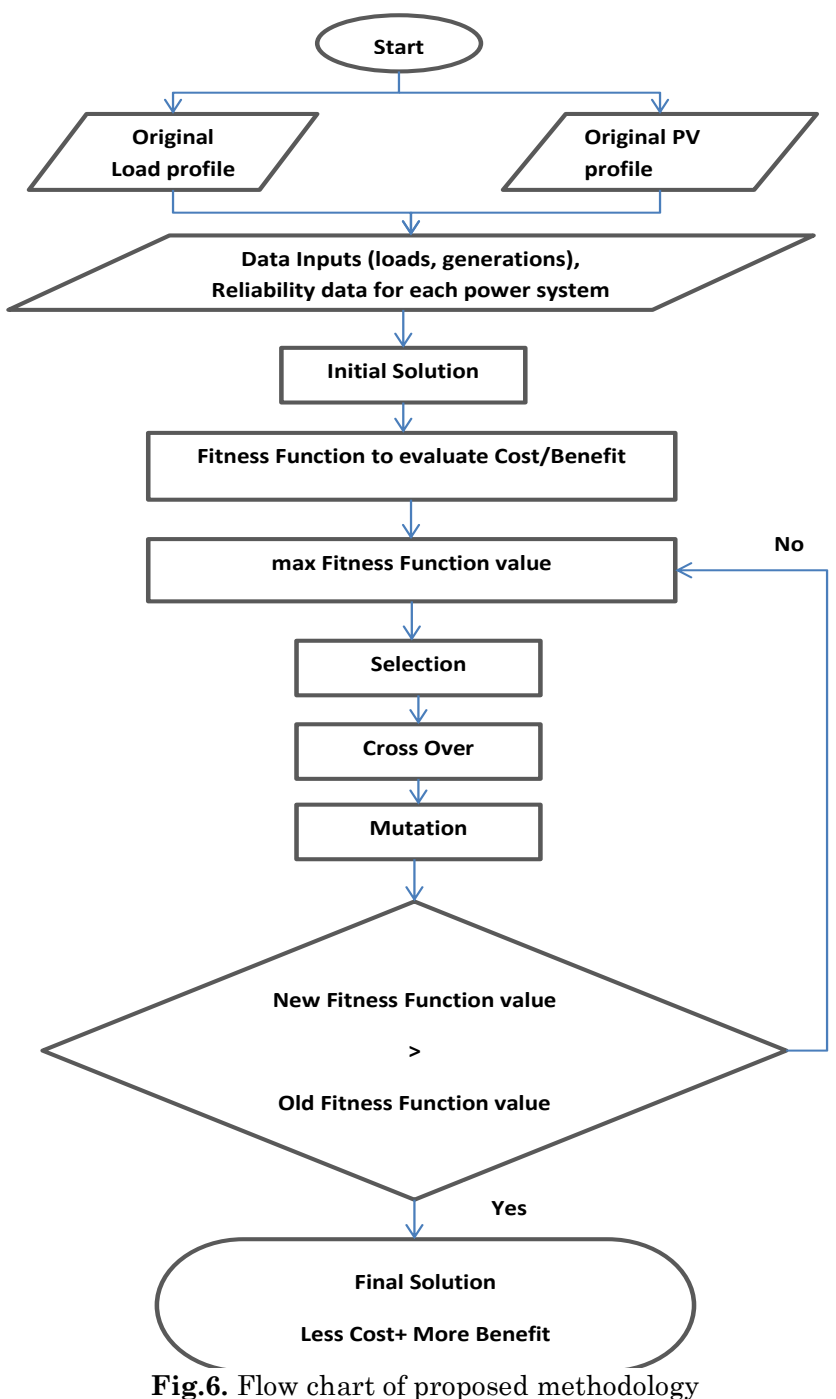

Fig.6. Flow chart of proposed methodology

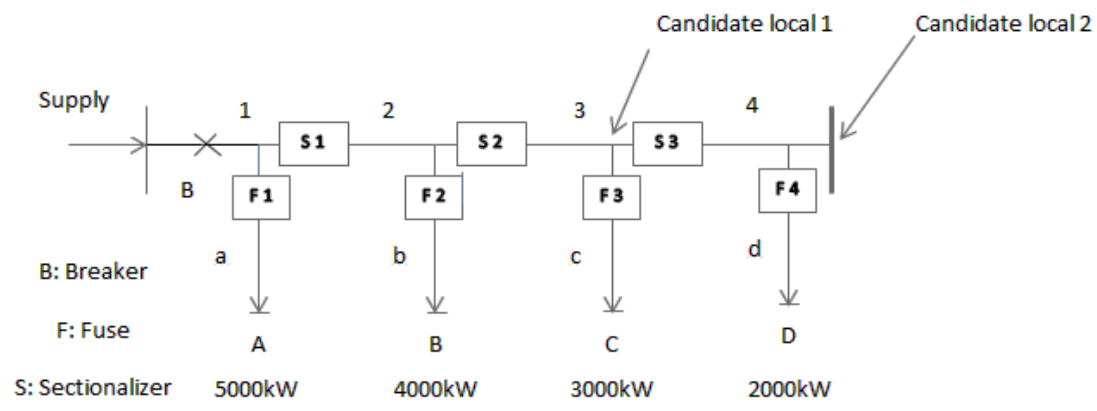

Fig. 7. Test System

\section{e. $\quad$ Process}

Genetic Algorithm iterates until it gets an optimal solution in specified limits. First of all, an initial population is developed through input technical data. Population size will remain constant throughout the process. For each chromosome, fitness function value in terms of cost and benefits is determined. The fitness function value as well as benefits are required to be maximized. Selection, crossover and mutation in each iteration help in the optimality of solutions. Parent chromosomes are selected from population based on fitness function value to produce the next generation. Cross over is done to explore new search regions in solution space. Mutation brings global solution. After these genetic operators, the fitness function is evaluated for the new generation. New fitness function values are compared with the old ones. If new fitness function value is better than the older one in specified limits, then it is required optimal solution. Otherwise, a new fitness function value is selected from original system and complete iteration process is repeated, until required optimality is obtained. Complete iterative process can be summarized through flow chart given in Fig. 6. 
Table 1

DG Features (Borges \& Falcao, 2006)

\begin{tabular}{cccc}
\hline DG types & $\begin{array}{c}\text { Total Cost } \\
{[£]}\end{array}$ & $\begin{array}{c}\text { Capacity } \\
{[\mathrm{kW}]}\end{array}$ & $\begin{array}{c}\text { Nominal } \\
\text { voltage } \\
{[\mathrm{kV}]}\end{array}$ \\
\hline DG 1 & 1000 & 2000 & 13.8 \\
DG 2 & 1500 & 3000 & 13.8 \\
DG 3 & 2000 & 4000 & 13.8 \\
\hline
\end{tabular}

\section{Case study}

The methodology proposed in this paper is applied to a distribution system taken from RBT system in (Li, 2013). Four different scenarios of PV allocation are simulated to understand the impact on reliability of the network. However, the proposed methodology is applicable to any number of candidate local points.

Fig.7 shows a radial distribution network with four nodes. Arrows represent candidate locals and boxes are protective devices of system. Table 1 gives DG cost data (Borges \& Falcao, 2006). Case studies consider the following system parameters for simulation purposes; Switching time of DG breaker is $0.5 \mathrm{~h}$ and losses cost is considered $\$ 100 / \mathrm{kW}$. The failure rate is considered as 0.0009 for each component.

This study considers four different cases where each case refers to different interconnection point of the DG. The first case considers DG allocation on node 2; the second case considers DG on node 3 , whereas node 4 is taken in case three. Calculation of the reliability indices for these cases is shown in Fig.8.

Fig.8. shows results of reliability analysis for different network configurations and their effects on reliability improvements. In the first case, DG is connected to node 2 and system wide randomness in SIAFI and SAIDI is observed that caused by many protective devices. This trend can be observed in other cases for node 3 and node 4 but there is a significant improvement in randomness. It can be said that protective devices produce more randomness and DG has the advantage that it is always connected to load points. The number of protective devices required to protect the transmission of electricity from point of generation to load is significantly decreased with the direct integration of DG near load points. This improves the reliability of the distribution network with integration of DG with less random behavior of SIFI and SAIDI. However, it is pertinent to mention that there are no significant improvements in interruptions duration.

An in-depth analysis of EENS (Expected Energy not supplied) reveals benefits of optimal locations of DG in the network. The system has high expected energy loss on node 2 because of many components being vulnerable to failure. Node 3 has less energy lost as compared to node 2. Whereas, node 4 has the least amount of energy lost accounts to that there is only one component. Hence, reliability can be improved by installing generation near to load points.

The final case i.e. case 4 includes the optimization of the DG allocation to determine the impacts on reliability of the system after selection of optimal DG interconnection point in a redial network. System reliability is determined under three different scenarios named before DG, after DG and after DG optimization. First scenario is simple radial system without DG installation, whereas second scenario is radial system with DG. While in the last scenario reliability for optimized DG allocation is determined.
(b11)Node 2

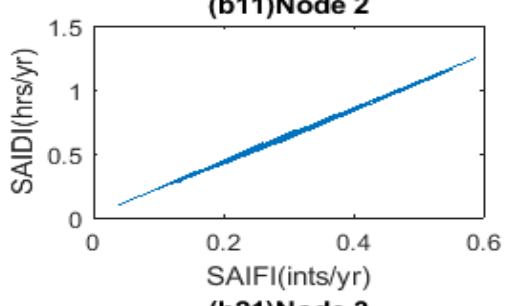

(b21)Node 3
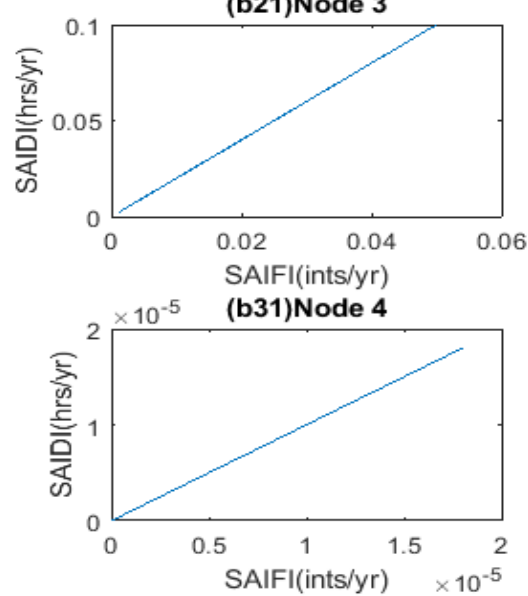

(b12)Node 2

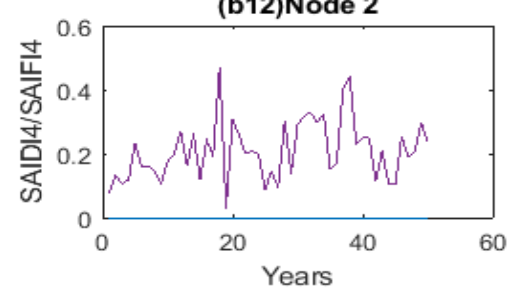

(b22)Node 3

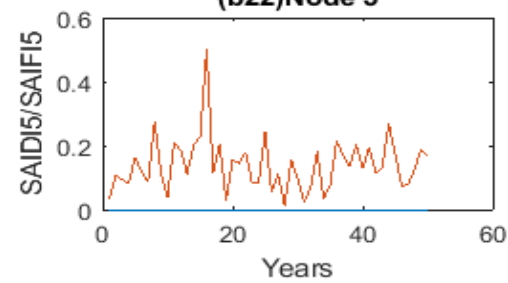

(b32)Node 4

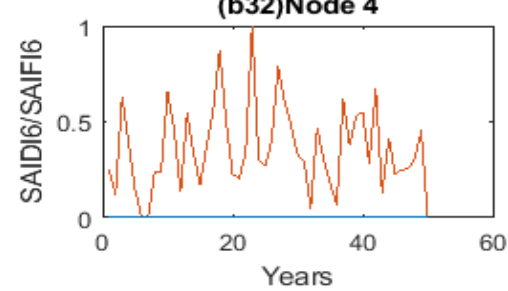

(b13)Node 2

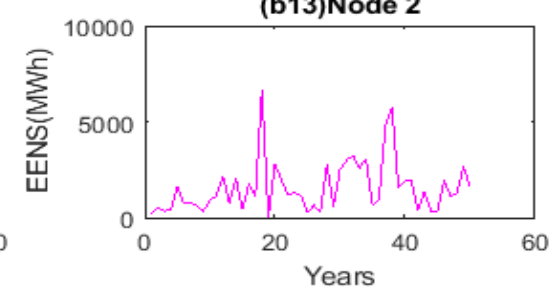

(b23)Node 3
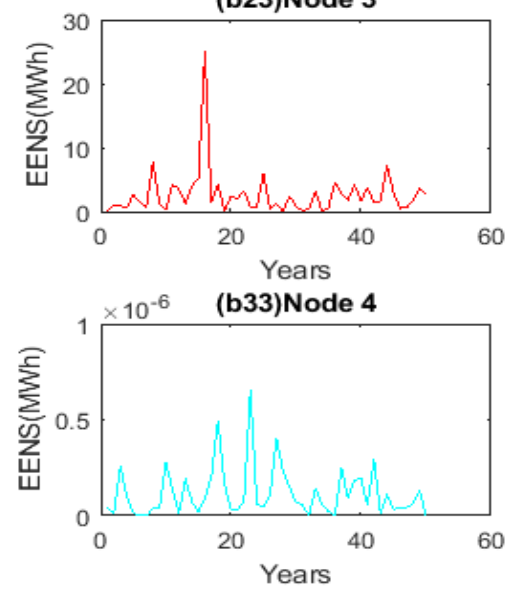

Fig. 8. Reliability analysis at various nodes of network 

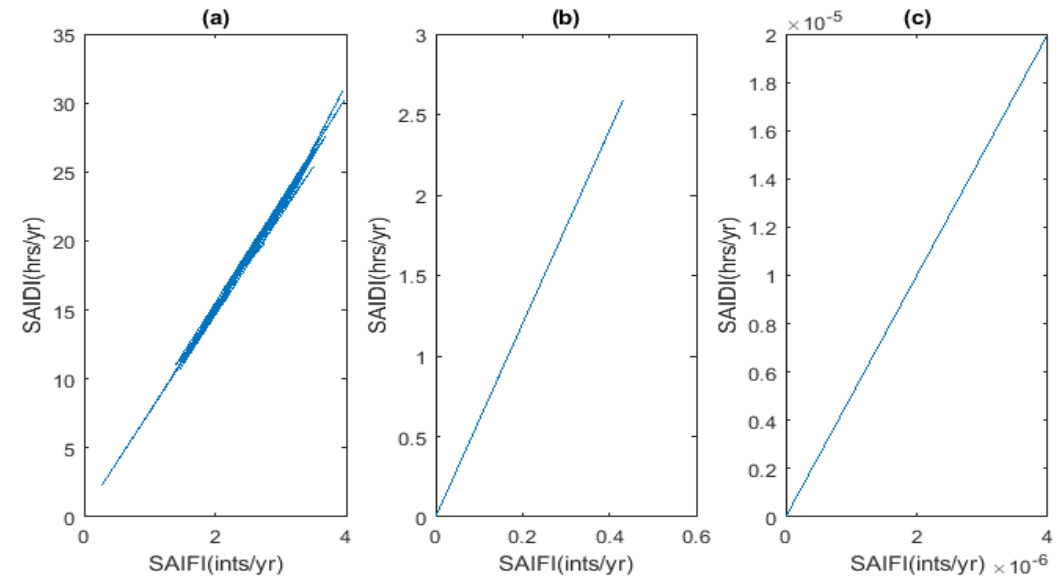

Fig. 9. Relationship of SAIFI and SAIDI

(a) Before DG (b) After DG (c) After DG Optimization.

Fig. 9 shows the relationship of reliability indices such as SAIFI and SAIDI in three different scenarios. The first one belongs to the network before DG, which has a higher number of interruptions and longer duration of these interruptions. It has an increasing trend with some randomness that is the result of widespread effects of some components failures close to the generation. It can be explained as in the case of breaker failure, supply to the whole radial system is cut off and a large portion is affected.

Fig. 9b shows the relationship of SAIFI and SAIDI after DG installation. The result reveals a large improvement in reliability indices. Both indices have much smaller values than the original system. It suggests that there are fewer numbers and duration of interruptions due to DG power supply in the event of main supply shut down. Moreover, it has smoother behavior as DG is always installed near customer load points and components failures have only local effects. The third one shows the relationship of both reliability indices after DG optimization using genetic algorithm. It has smoother increasing trend resulting from optimal DG locations and least outage effects on system. It shows double benefits as compared to the original system. It clearly shows that optimality of DG locations in the network is as important as its DG allocation for reliable supply.
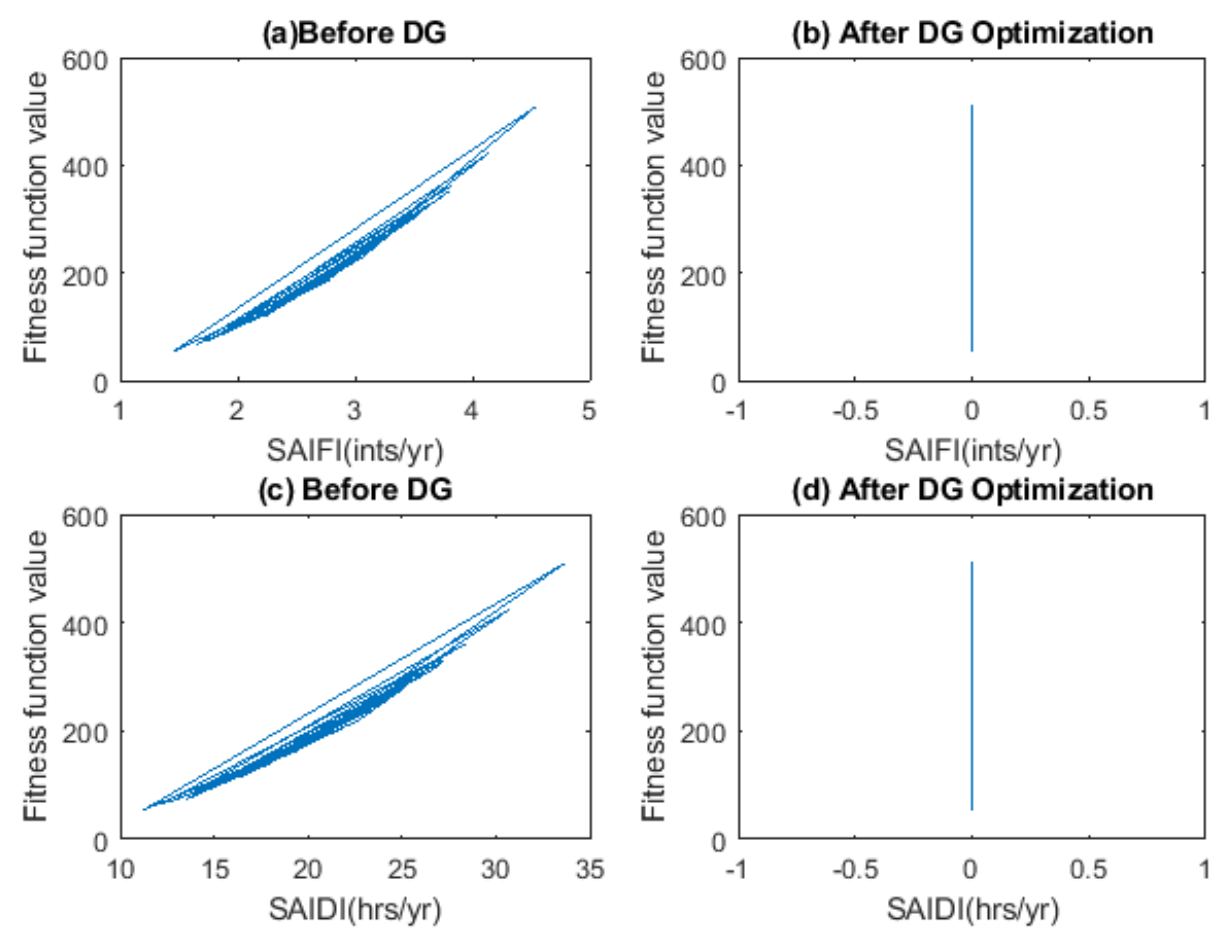

Fig 10. Effects of SAIFI and SAIDI on fitness function values 
(a)

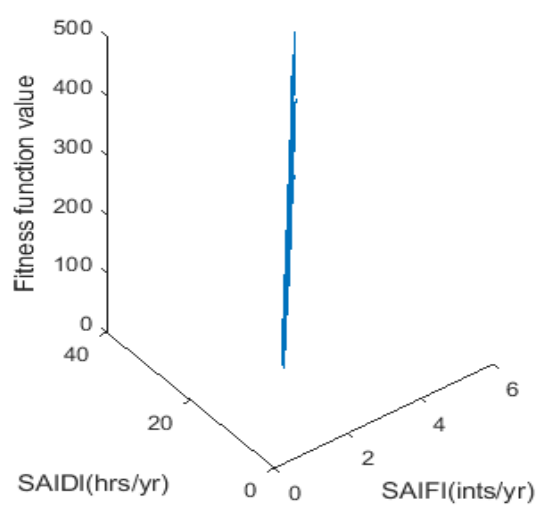

(b)

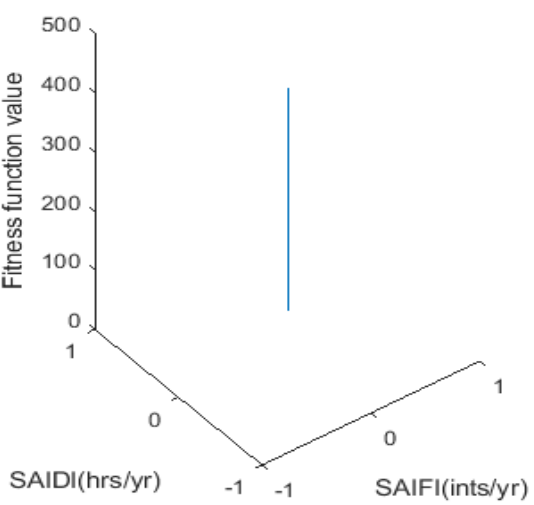

Fig. 11. Combined effects of SAIFI and SAIDI on fitness function values (a) Before DG (b) After DG Optimization.
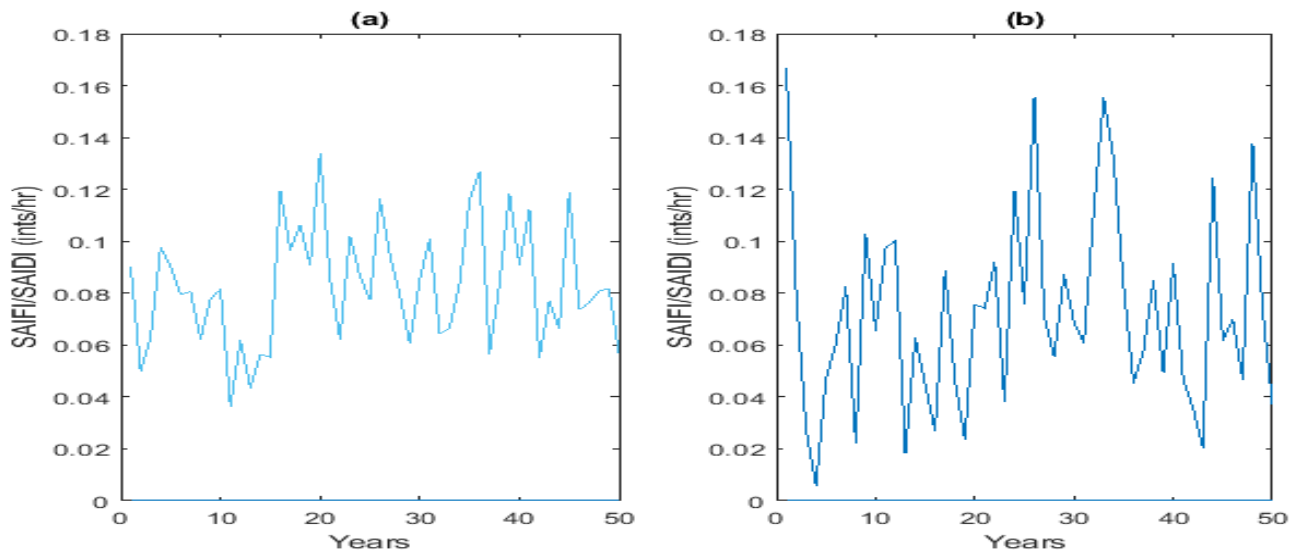

Fig. 12. Average hourly interruptions in a year (a) Before DG (b) After DG.
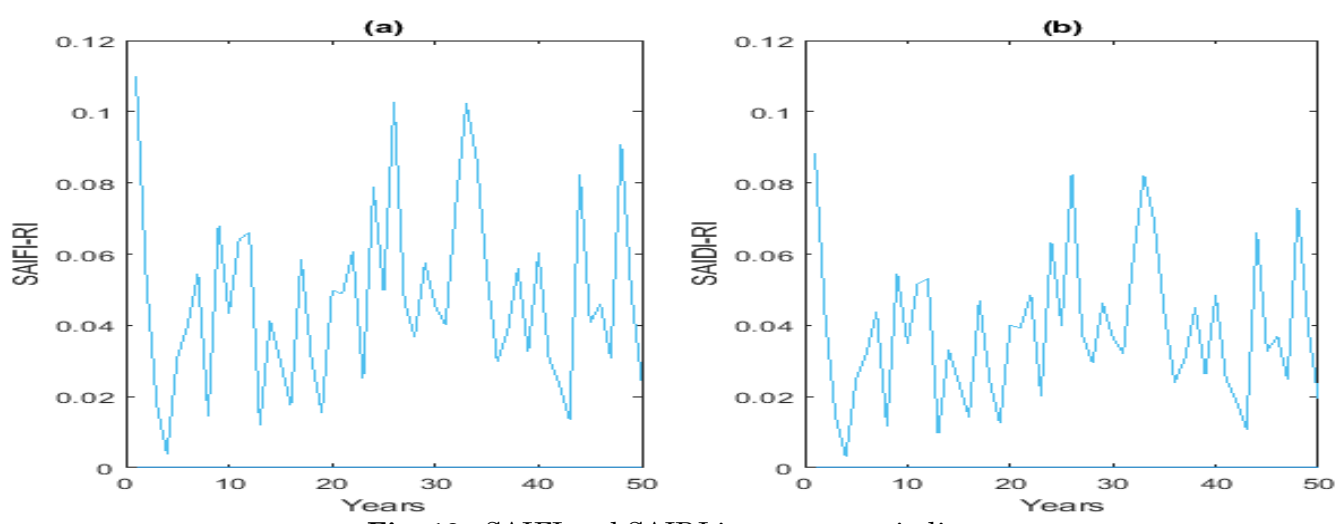

Fig. 13. SAIFI and SAIDI improvement indices

(a) SAIFI reduction index (b)SAIDI reduction index

The effects of SAIFI and SAIDI on fitness function before and after the DG optimization are described in Fig 10. Fitness function value increases as SAIFI and SAIDI values increase. This is due to the increment of power losses with increasing values of SAIFI and SAIDI. Randomness can be attributed to widespread effects of components failures. After DG optimization, SAIFI and SAIDI have zero values due to continuous supply by DG.
However, increment in fitness function value can be attributed to a continuous reduction in power losses after DG allocation in the optimization process. Both reliability indices have similar combined effects on fitness function values before DG and after DG optimization as described in Fig 11.

The average hourly number of interruptions per year is showed in Fig.12 for system with and without DG 
installed. There are slight improvements in the interruptions after DG allocation without optimization. It highlights importance of optimization in planning. As network still has components, which can be out of order, but they are lesser in number as compared to original network.

Fig 13 shows improvements in the values of both reliability indices SAIFI and SAIDI. Reduction in the number of interruptions and their duration is described by these indices after DG installation. These indices play an important role in determining reliability improvements of networks. Total expected energy lost per year is showed in Fig. 14 for three different scenarios. In the network without DG, total energy lost in a year is much higher. Long radial system and more components vulnerable to failure are the main causes of this fact. After DG, there is a vast improvement in total supply outage in a year and it is very close to zero. Optimization brings total supply outage in a year to zero supply outage with vast economic benefits. It represents a completely reliable system with continuous supply and no outages.

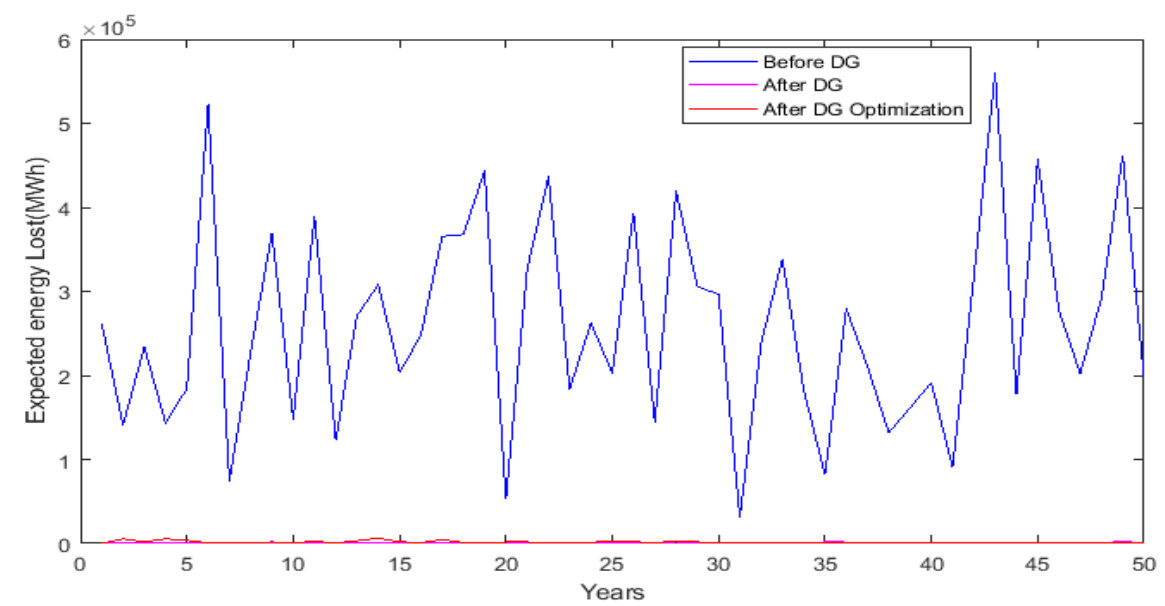

Fig 14. Total expected energy lost per year.
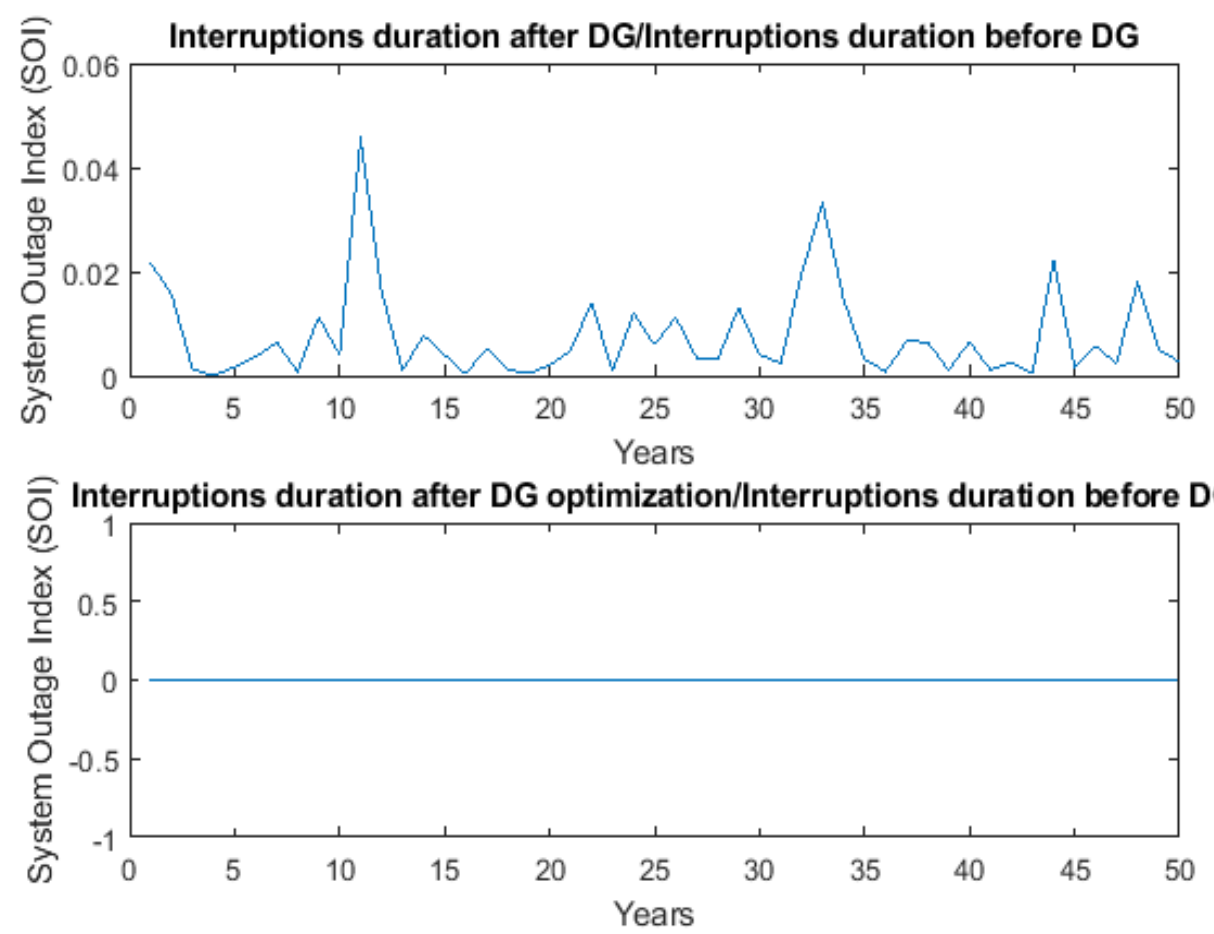

Fig 15. System outage index 


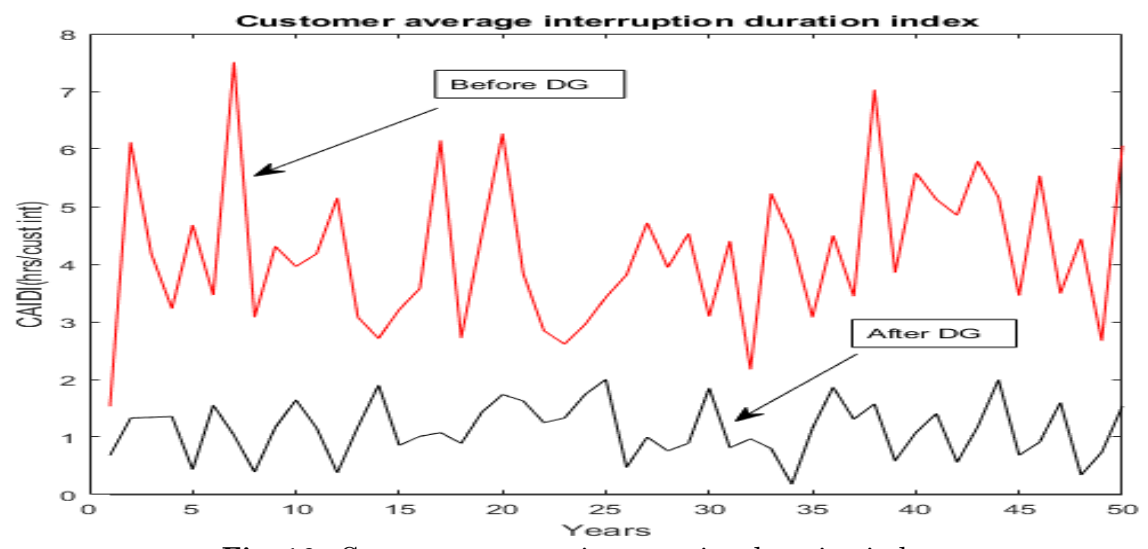

Fig. 16. Customer average interruption duration index.

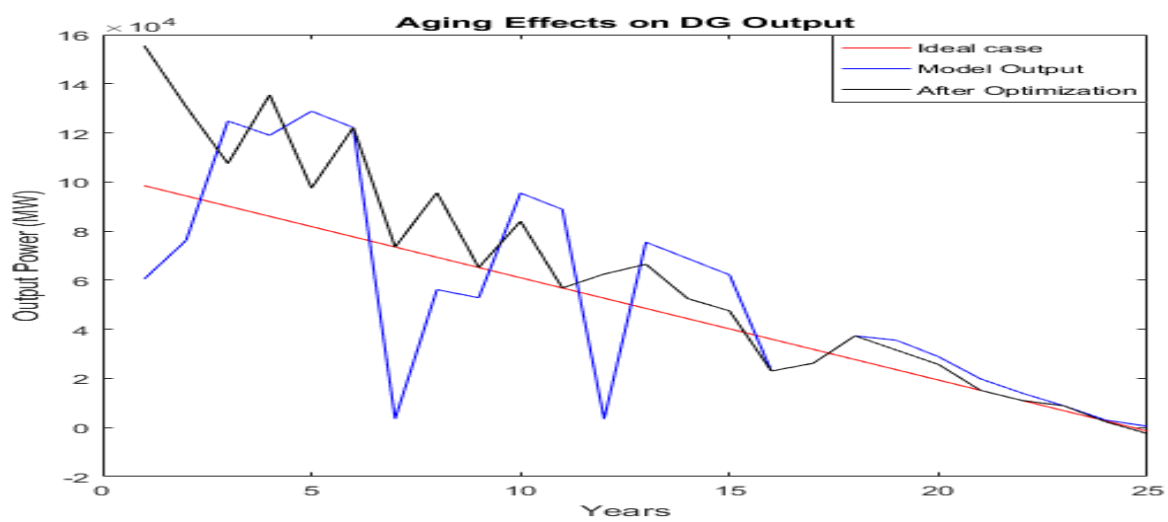

Fig 17. Aging effects on DG outputs

System outage index is showed in Fig. 15 for two different scenarios. The first one describes a vast improvement in total outage hours after DG allocations. It is near to zero, which means that there is a very small probability of supply outage. The second one represents the advantages of optimal DG allocations. If DG has an optimal location, the probability of supply outage is zero.

Fig. 16 presents average interruption duration for the network with and without DG allocations. There is an improvement in supply outage hours suffered by the customer after DG allocations. It cannot be zero as the probability of failures of protective devices is not possible to be zero.

Aging effects on the DG output are summarized for 25 years life span of PV system as given in Fig.17. The ideal output has straight line i.e. a constant aging effect. Mathematical modeled aging effects after DG have large variations from the ideal or original case. However, after the optimization process, mathematical model is closer to the ideal case. It shows the advantages of implementing the genetic algorithm for optimization purposes.

Reliability of the optimized network is much higher than single node DG allocation. Although, the reliability of network in the first three cases is better than the original system, but they present the same problems as system-wide effects of components failures. The system still has load points far away from generation and components are vulnerable to failures, however in final case generation is as much close to load points as possible with optimization. It has far more advantages than single node DG allocation in terms of system reliability and supply continuity.

\section{Conclusion}

This paper presented a method for composition assessment of distribution system by deciding optimal location and sizing of DG to increase benefits and reduce losses. A reliability test system is used to validate the applicability of the proposed methodology in the distribution network. The results suggest that the randomness in the reliability indices can be reduced to make the system more reliable by appropriate allocation of the DG using optimization technique such as GA. Components close to generation have widespread effects on the radial system and they produce some randomness. Closer allocation of DG to the load points reduces randomness as it has only local effects of components failures. The reduction in power transmission protective equipment is obtained with DG installations near load points, can potentially improve power system reliability. Optimal allocation and sizing of DG can double the benefits in terms of reduced losses and increased reliability. The proposed approach can benefit power system planners in the selection of appropriate location and size of the DG considering the penetration level of the DG to enhance network reliability.

\section{References}

Ahadi, A., Ghadimi, N., \& Mirabbasi, D. (2014). Reliability assessment for components of large scale photovoltaic systems. Journal of Power Sources, 264, 211-219. 
Altamimi, A., \& Jayaweera, D. (2017). Reliability performances of grid-integrated PV systems with varying climatic conditions. In IET International Conference on Resilience of Transmission and Distribution Networks (RTDN 2017), Birmingham, UK, 2017. IET Publishing.

Bodenhofer, U. (2003). Genetic algorithms: theory and applications. Lecture notes, Fuzzy Logic Laboratorium Linz-Hagenberg, Winter.

Borges, C. L. T., \& Falcao, D. M. (2006). Optimal distributed generation allocation for reliability, losses, and voltage improvement. International Journal of Electrical Power \& Energy Systems, 28(6), 413-420.

Bouktir, T., Slimani, L., \& Belkacemi, M. (2004). A genetic algorithm for solving the optimal power flow problem. Leonardo Journal of Sciences, 4, 44-58.

Calais, M., Myrzik, J., Spooner, T., \& Agelidis, V. G. (2002). Inverters for single-phase grid connected photovoltaic systems-an overview. In Power Electronics Specialists Conference, 2002. pesc 02. 2002 IEEE 33rd Annual (Vol. 4, pp. 1995-2000). IEEE.

Celli, G., Ghiani, E., Mocci, S., \& Pilo, F. (2005). A multiobjective evolutionary algorithm for the sizing and siting of distributed generation. IEEE Transactions on Power Systems, 20(2), 750-757.

Chen, P.-H., \& Chang, H.-C. (1995). Large-scale economic dispatch by genetic algorithm. IEEE Transactions on Power Systems, 10(4), 1919-1926.

Dellosa, J. (2016). Potential Effect and Analysis of High Residential Solar Photovoltaic (PV) Systems Penetration to an Electric Distribution Utility (DU). Int. Journal of Renewable Energy Development, 5(3),179-185, doi: 10.14710/ijred.5.3.179-185

Eltawil, M. A., \& Zhao, Z. (2010). Grid-connected photovoltaic power systems: Technical and potential problems-A review. Renewable and Sustainable Energy Reviews, 14(1), $112-129$.

Gerbex, S., Cherkaoui, R., \& Germond, A. J. (2001). Optimal location of multi-type FACTS devices in a power system by means of genetic algorithms. IEEE Transactions on Power Systems, 16(3), 537-544.

Goyal, V., \& Mahapatra, S. (2011). Application of genetic algorithm in the optimum placement of distributed generator in distributed power system. International Journal of Computer Applications, 30(6), 1-5.

Grigg, C., Wong, P., Albrecht, P., Allan, R., Bhavaraju, M., Billinton, R., Chen Q., Fong C., Haddad S., Kuruganty S., Mukerji R., Li, W., Patton D., Rau N., Reppen D., Schneider A., Shahidehpour M., \& C. Singh (1999). The IEEE reliability test system-1996. A report prepared by the reliability test system task force of the application of probability methods subcommittee. IEEE Transactions on power systems, 14(3), 1010-1020.

International Energy Agency. (2017). Trends 2017 in Photovoltaic Applications Executive Summary. Retrieved from http://www.ieapvps.org/fileadmin/dam/public/report/statistics/IEAPVPS_-Trends_in_PV_Applications_2017_. _EXECUTIVE_SUMMARY.pdf

Jantsch, M., Real, M., Häberlin, H., Whitaker, C., Kurokawa, K., Blässer, G., Kremer, P \& Verhoeve, C. W. G. (1997). Measurement of PV maximum power point tracking performance. Netherlands Energy Research Foundation ECN. Retrieved from http://citeseerx.ist.psu.edu/viewdoc/download?doi=10.1.1.6 $21.4660 \&$ rep $=$ rep $1 \&$ type $=$ pdf

Jayaweera, D., \& Islam, S. (2014). Security of energy supply with change in weather conditions and dynamic thermal limits. IEEE Transactions on Smart Grid, 5(5), 2246-2254.

Kishore, L. N., \& Fernandez, E. (2011). Reliability well-being assessment of PV-wind hybrid system using Monte Carlo simulation. In Emerging Trends in Electrical and Computer Technology (ICETECT), 2011 International Conference on (pp. 63-68). IEEE.

Li, W. (2013). Reliability assessment of electric power systems using Monte Carlo methods. Springer Science \& Business Media.

Premkumar, M., Karthick, K and Sowmya, R(2018). A Review on Solar PV Based Grid Connected Microinverter Control Schemes and Topologies. Int. Journal of Renewable Energy Development, 7(2),171-182, doi.org/10.14710/ijred.7.2.171182

Puttgen, H. B., Macgregor, P. R., \& Lambert, F. C. (2003). Distributed generation: Semantic hype or the dawn of a new era? IEEE Power and Energy Magazine, 99(1), 22-29.

Quezada, V. H. M., Abbad, J. R., \& Roman, T. G. S. (2006). Assessment of energy distribution losses for increasing penetration of distributed generation. IEEE Transactions on Power Systems, 21(2), 533-540.

Rodríguez-Gallegos, C. D., Gandhi, O., Bieri, M., Reindl, T., \& Panda, S. K. (2018). A diesel replacement strategy for offgrid systems based on progressive introduction of PV and batteries: An Indonesian case study. Applied Energy, 229, 1218-1232.

Rodríguez-Gallegos, C. D., Gandhi, O., Yang, D., AlvarezAlvarado, M. S., Zhang, W., Reindl, T., \& Panda, S. K. (2017). A siting and sizing optimization approach for PVbattery-diesel hybrid systems. IEEE Transactions on Industry Applications, 54(3), 2637-2645.

Rodríguez-Gallegos, C. D., Yang, D., Gandhi, O., Bieri, M., Reindl, T., \& Panda, S. K. (2018). A multi-objective and robust optimization approach for sizing and placement of PV and batteries in off-grid systems fully operated by diesel generators: An Indonesian case study. Energy, 160, 410429.

Rújula, A. A. B., Amada, J. M., Bernal-Agustin, J. L., Loyo, J. M. Y., \& Navarro, J. A. D. (2005). Definitions for distributed generation: a revision. In International Conference on Renewable Energy and Power Quality March (pp. 16-18).

Stember, L. H., Huss, W. R., \& Bridgman, M. S. (1982). A methodology for photovoltaic system reliability \& economic analysis. IEEE Transactions on Reliability, 31(3), 296-303.

Walters, D. C., \& Sheble, G. B. (1993). Genetic algorithm solution of economic dispatch with valve point loading. IEEE Transactions on Power Systems, 8(3), 1325-1332.

Wang, Y., Zhang, P., Li, W., \& Kan'an, N. H. (2012). Comparative analysis of the reliability of grid-connected photovoltaic power systems. In Power and Energy Society General Meeting, 2012 IEEE (pp. 1-8). IEEE.

Zhang, P., Li, W., Li, S., Wang, Y., \& Xiao, W. (2013). Reliability assessment of photovoltaic power systems: Review of current status and future perspectives. Applied Energy, $104,822-833$

Zulu, E. \& Jayaweera, D. (2014). Reliability assessment in active distribution networks with detailed effects of PV systems. Journal of Modern Power Systems and Clean Energy, 2(1), $59-68$. 